\title{
Insegurança alimentar e indicadores antropométricos, dietéticos e sociais em estudos brasileiros: uma revisão sistemática
}

\author{
Food insecurity and anthropometric, dietary and social indicators \\ in Brazilian studies: a systematic review
}

Dayane de Castro Morais ${ }^{1}$

Luiza Veloso Dutra ${ }^{1}$

Sylvia do Carmo Castro Franceschini ${ }^{1}$

Silvia Eloiza Priore ${ }^{1}$

' Departamento de Nutrição e Saúde, Universidade Federal de Viçosa. Av. P.H. Rolfs s/n - Campus Universitário, Centro. 36.570-900 Viçosa MG Brasil.

dayanecm@yahoo.com.br
Abstract The scope of this systematic review was to relate food insecurity, detected using the Brazilian Food Insecurity Scale (EBIA), with anthropometric, dietary and social indicators. The search was conducted in electronic databases (ScieLO, LILACS, MEDLINE), with a selection of studies by titles and abstracts, and later full reading. Studies identified in bibliographic references were included. Of the 215 reviewed, 15 fulfilled inclusion criteria (association between insecurity and anthropometric, dietary or social indicators, detected by the EBIA), whereby three had more than one variable of interest. A relationship was observed between food insecurity and height/age and weight/age of child indices, as well as obesity in women. Lower consumption of regulating, tissue-building food products and iron, and higher carbohydrate intake are associated with food insecurity. There was a relationship between social indicators, such as lower income and education, lack of employment and basic sanitation. The EBIA was associated in some studies with nutritional and social indicators, but should be used in conjunction with other tools in order to cover the multiple dimensions of food and nutrition security.

Key words Food and nutrition security, Nutritional status, Food consumption, Social indicators
Resumo Objetivou-se nesta revisão sistemática relacionar situação de insegurança alimentar, detectada pela Escala Brasileira de Insegurança Alimentar (EBIA), com indicadores antropométricos, dietéticos e sociais. Realizou-se busca em bases eletrônicas (ScieLO, LILACS, MEDLINE), com seleção dos estudos pelos títulos e resumos, e posteriormente por leitura integral. Incluiu-se estudos identificados nas referências bibliográficas. Dos 215 revisados, 15 contemplaram critérios de inclusão (associação entre indicadores antropométricos, dietéticos ou sociais com insegurança alimentar, detectada pela EBIA), sendo que três contemplaram mais de uma variável de interesse. Observou-se relação entre insegurança alimentar e indices estatura/idade e peso/idade em crianças, e para obesidade em mulheres. Menor consumo de alimentos reguladores, construtores e ferro, e maior consumo de carboidratos associou-se à insegurança alimentar. Houve relação entre indicadores sociais, como menor renda e escolaridade, ausência de vínculo empregatício e saneamento básico. A EBIA apresentou associação, em alguns estudos, com indicadores nutricionais $e$ sociais, mas deve ser utilizada conjuntamente com outros instrumentos, visando abranger múltiplas dimensões da segurança alimentar e nutricional. Palavras-chave Segurança alimentar e nutricional, Estado nutricional, Consumo de alimentos, Indicadores sociais 


\section{Introdução}

No Brasil, a segurança alimentar e nutricional (SAN) é definida por lei como a garantia de acesso regular e permanente a alimentos de qualidade, em quantidade suficiente, sem comprometer $o$ acesso a outras necessidades essenciais e que respeitem a diversidade cultural, sendo ambiental, cultural, econômica e socialmente sustentáveis ${ }^{1}$ e tendo como princípios básicos o direito humano à alimentação adequada e à soberania alimentar ${ }^{2}$.

O conceito de segurança alimentar e nutricional foi ampliado, ultrapassando o marco dos processos de carências nutricionais ${ }^{3}$, em função da transição nutricional marcada pela dupla carga de doenças, ou seja, a coexistência das carenciais (desnutrição, e deficiências de micronutrientes) e das relacionadas ao excesso de peso e associadas às crônicas não transmissíveis ${ }^{4}$. Neste contexto, a (in)segurança alimentar pode ser detectada por diversas manifestações, desde fome, desnutrição e carências específicas como também pelo excesso de peso e doenças decorrentes da alimentação inadequada $^{5}$, que geralmente associam-se a outro tipo de fome, a "fome oculta" que caracteriza-se pela inadequação alimentar quantitativa e/ou qualitativamente ${ }^{6,7}$, resultando em prejuízos no desempenho cognitivo, capacidade de trabalho e morbimortalidades associadas ${ }^{8}$.

Para mensurar a (in)segurança alimentar, desenvolveu-se e validou-se, a Escala Brasileira de Insegurança Alimentar (EBIA), a partir da experiência da Universidade de Cornell ${ }^{5}$. Para a sua validação no Brasil, foi realizado um estudo multicêntrico, apoiado pela Organização PanAmericana de Saúde 9 , visando contemplar diferentes contextos sociais, econômicos e culturais, no meio urbano e rural, para que uma única escala pudesse ser utilizada no país ${ }^{10}$. O processo de validação se deu em duas etapas: uma qualitativa e outra quantitativa, resultando em um instrumento de mensuração de insegurança alimentar com solidez satisfatória (com $\alpha$ de Chronbach variando de 0,91 a 0,94 ), mais condizente com a realidade brasileira e capaz de identificar famílias em situação de vulnerabilidade social que necessitam de intervenções ${ }^{11}$.

A EBIA é um método que investiga de forma direta a (in)segurança alimentar das famílias ${ }^{5}$, sendo composta por 14 perguntas fechadas, relacionadas à percepção dos entrevistados sobre a situação alimentar nos últimos três meses ${ }^{12}$.

Nesta escala a questão alimentar é percebida em seus vários aspectos, incluindo os psicológi- cos, como a preocupação com o comprometimento da qualidade e quantidade da alimentação da família ${ }^{5}$. A classificação é dependente da presença de menores de 18 anos na família, sendo a escala respondida em sua totalidade nessa situação ${ }^{13}$.

A (in)segurança alimentar e nutricional também pode ser avaliada por indicadores nutricionais e socioeconômicos. Medidas antropométricas são indicadores importantes do estado nutricional, mas avaliam de forma indireta e limitante a segurança alimentar e nutricional, já que podemos encontrar indivíduos em segurança alimentar que estejam abaixo ou acima do peso ideal. Esta situação indica comprometimento da qualidade da dieta ${ }^{5,14}$. O estado nutricional e o acesso aos alimentos são frequentemente utilizados como fatores determinantes de (in)segurança alimentar e nutricional, mas bom estado nutricional e ausência de fome não são garantia suficiente de atingir a segurança alimentar e nutricional ${ }^{15}$.

A investigação do consumo alimentar individual é capaz de detectar a situação de insegurança alimentar, já que permite conhecer a ingestão alimentar, em seus aspectos qualitativos e quantitativos ${ }^{16}$.

Os indicadores socioeconômicos também são uma forma de mensurar a (in)segurança alimentar já que domicílios com acesso precário a renda, bens e serviços estão mais vulneráveis a esta situação e às repercussões da mesma, como alterações nutricionais e no desenvolvimento psicomotor $^{11,17}$.

Vale ressaltar que nenhum indicador, isoladamente, consegue abranger as múltiplas dimensões da segurança alimentar e nutricional ${ }^{5}$, uma vez que cada método monitora o fenômeno segundo ótica própria, tornando os mesmos complementares ${ }^{16}$.

Este estudo objetivou relacionar a situação de (in)segurança alimentar, determinada pela Escala Brasileira de Insegurança Alimentar, com os indicadores antropométricos, dietéticos e sociais, em diferentes populações e faixas etárias, em estudos brasileiros.

\section{Metodologia}

Inicialmente realizou-se uma busca sistemática em bases eletrônicas de dados (ScieLO, Lilacs, Medline), incluindo os estudos publicados nos últimos 9 anos, visando uma abordagem da situação de (in)segurança alimentar detectada pela EBIA, já que este instrumento foi validado no 
país em 2004. Os descritores utilizados foram segurança alimentar e nutricional, estado nutricional, consumo de alimentos, indicadores sociais e Escala Brasileira de Insegurança Alimentar, bem como seus respectivos termos em inglês (food security, nutritional status, food consumption, social indicators, association e Brazilian Scale of Food Insecurity) e em espanhol (la seguridad alimentaria, estado nutricional, consumo de alimento, indicadores sociales, asociación e Comida Brasileña Escala de Inseguridad).

Incluiu-se nesta revisão sistemática artigos originais, de cunho nacional, que apresentavam associação entre (in)segurança alimentar, detectada pela EBIA, e algumas das variáveis de interesse neste estudo (estado nutricional, consumo de alimentos e indicadores sociais). Não foram incluídos artigos de revisão, monografias, dissertações, teses, capítulos e livros, além de estudos realizados em outros países, já que padronizou-se como instrumento de detecção de (in)segurança alimentar, neste estudo, a EBIA, escala esta validada para o Brasil. Não foram impostos limites para o idioma da publicação, idade ou gênero da unidade de estudo.

Para a elaboração da revisão sistemática primeiramente realizou-se busca dos descritores nas bases de dados já descritas, identificando 215 estudos publicados no período de interesse. A etapa seguinte constou de revisão e seleção dos estudos, avaliando primeiramente os títulos, na qual foram excluídos 175 documentos, sendo 29 por serem repetidos. Aos 40 artigos originais selecionados para a leitura dos resumos, foram acrescidos 18 estudos obtidos a partir da revisão das referências bibliográficas dos artigos selecionados nesta etapa, com o intuito de identificar estudos de potencial relevância, não identificados na busca eletrônica e ainda não incluídos na revisão sistemática, que atendiam aos critérios de inclusão.

Deste total de 58 artigos, 38 foram excluídos, sendo 27 por não relatar associação de (in)segurança alimentar com as variáveis de interesse neste estudo e 11 por não terem sido realizados no Brasil. Dos 20 artigos restantes, 5 foram excluídos, após leitura integral dos mesmos, por não relataram associação entre (in)segurança alimentar e as variáveis de interesse neste estudo. Assim, 15 estudos contemplaram os critérios de inclusão e foram utilizados nesta revisão sistemática (Figura 1).

\section{Resultados}

Dentre os estudos selecionados $(n=15)$, houve relato de associação entre (in)segurança alimentar e estado nutricional, a partir de dados antropométricos $(\mathrm{n}=6)$; (in)segurança alimentar e consumo de alimentos $(\mathrm{n}=4)$; e entre (in)segurança alimentar e indicadores sociais $(n=8)$. Destaca-se que três dos estudos abordaram mais de uma variável de interesse, sendo incluídos então em mais de um quadro de análise.

No Quadro 1 estão descritas as associações entre o estado nutricional, avaliado por dados antropométricos (peso e altura), e a presença de insegurança alimentar domiciliar. Observa-se que, quando os indivíduos da amostra eram crianças, a relação entre estas variáveis apresentou maior magnitude com o índice estatura/idade (E/ I ${ }^{12,18}$ e com o índice peso/idade $(\mathrm{P} / \mathrm{I})^{19}$.

Não houve associação entre variáveis de estado nutricional e insegurança alimentar apenas no estudo de Oliveira et al. ${ }^{20}$ cuja unidade amostral foi de adolescentes e adultos, do Nordeste brasileiro.

Nas avaliações entre o consumo de alimentos e a (in)segurança alimentar (Quadro 2), foi relatado menor consumo de alimentos construtores e reguladores, entre indivíduos em algum grau de insegurança alimentar domiciliar ${ }^{21-23}$. Observa-se consumo excessivo de glicídios entre as crianças e famílias em insegurança alimen$\operatorname{tar}^{22,23}$. Observou-se que crianças que viviam em domicílios em insegurança alimentar apresentaram consumo inadequado de energia e deficiente em ferro, sendo esta diferença significante ${ }^{21,23}$ Verificou-se maior omissão de refeições entre famílias em insegurança alimentar ${ }^{22}$. Não foram encontradas diferenças entre o consumo de vitaminas A e C e cálcio com a presença de insegurança alimentar domiciliar ${ }^{21,23}$.

Gomes e Gubert ${ }^{24}$, avaliando o consumo de leite materno em crianças menores de 2 anos, observaram associação entre a prática de aleitamento materno e a situação de insegurança alimentar domiciliar apenas para maiores de 12 meses. A condição de insegurança alimentar não se associou com a prática de aleitamento materno no primeiro ano de vida, bem como com a introdução precoce de alimentação complementar neste mesmo estudo.

Entre os estudos que relacionaram presença de (in)segurança alimentar e indicadores sociais (Quadro 3), todos apresentaram associação entre presença de insegurança alimentar e menor renda ${ }^{11,19,21,22,25-29}$, sendo que a maioria destes 


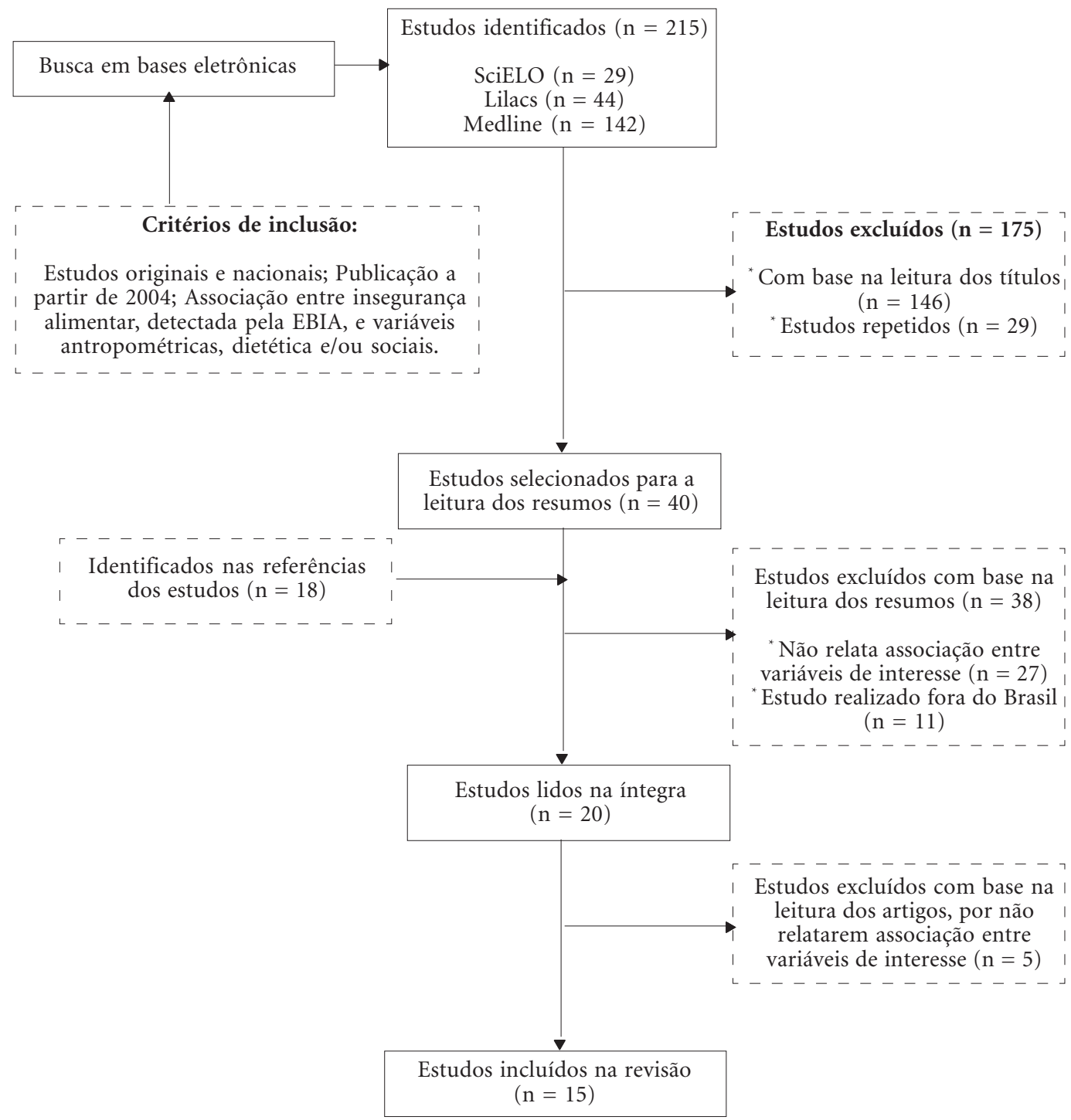

Figura 1. Etapas da elaboração da revisão sistemática.

também encontrou associação entre esta situação e menor escolaridade do responsável ou chefe da família ${ }^{11,19,25,26,27,30}$, inclusive menor escolaridade materna ${ }^{21,28}$. Observou-se também associação entre insegurança alimentar e maior número de moradores no domicílio ${ }^{21,27,28,30}$. A condição de insegurança alimentar mostrou-se associada à presença de moradores menores de 18 $\operatorname{anos}^{25,28}$.

Ainda sobre indicadores sociais, observou-se associação entre situação de insegurança alimentar domiciliar e condições inadequadas de sane- amento básico e material de construção da mo$\operatorname{radia}^{25,29}$, assim como com baixas condições de saneamento ${ }^{19}$. Verificou-se presença de insegurança alimentar em domicílios cujo informante autorreferiu raça/cor parda ou preta ao ser entrevistado ${ }^{25}$.

Encontrou-se associação entre presença de insegurança alimentar e ausência de vínculo empregatício do chefe da família ${ }^{27}$, bem como com a presença de maior número de filhos ${ }^{11,21}$. Houve maior situação de insegurança alimentar em famílias da zona rural ${ }^{26}$, e em domicílios sem pre- 
Quadro 1. Relação entre estado nutricional (dados antropométricos) e presença de insegurança alimentar domiciliar, detectada pela Escala Brasileira de Insegurança Alimentar (EBIA) nos estudos revisados.

\begin{tabular}{|c|c|c|c|c|c|}
\hline \multirow[b]{2}{*}{ Referência } & \multicolumn{3}{|c|}{ Metodologia } & \multicolumn{2}{|r|}{ Resultados } \\
\hline & $\begin{array}{l}\text { Unidade de } \\
\text { Estudo }\end{array}$ & Antropometria & $\begin{array}{l}\text { Avaliação } \\
\text { do EN }\end{array}$ & $\begin{array}{l}\text { Insegurança } \\
\text { Alimentar }\end{array}$ & Associação entre variáveis \\
\hline $\begin{array}{l}\text { Valásquez- } \\
\text { Melendez } \\
\text { et al., } \\
2011^{26}\end{array}$ & $\begin{array}{l}\text { Mulheres em } \\
\text { idade } \\
\text { reprodutiva, } \\
\text { participantes } \\
\text { da PNDS, } \\
2006 \\
(\mathrm{n}=10.226)\end{array}$ & $\begin{array}{l}\text { - Peso e } \\
\text { estatura, em } \\
\text { duplicata }\end{array}$ & - IMC $\left(\mathrm{OMS}^{31}\right)$ & $\begin{array}{l}\text { - 40,9\% dos } \\
\text { domicílios } \\
\text { em IA }\end{array}$ & $\begin{array}{l}\text { - Associação entre IA e obesidade, } \\
\text { sendo mais prevalente entre mulheres } \\
\text { em IAL ( } \mathrm{RP}=1,22 \text {; IC: } 1,04-1,44) \text { e } \\
\text { IAM ( } \mathrm{RP}=1,53 \text {; IC: } 1,22-1,93) \\
\text { - Prevalência maior de obesidade } \\
\text { (49\%) entre mulheres que vivem em } \\
\text { domicílios em IA ( } \mathrm{p}=0,01)\end{array}$ \\
\hline $\begin{array}{l}\text { Oliveira et } \\
\text { al. } 2010^{18}\end{array}$ & $\begin{array}{l}\text { Crianças } \\
\text { menores de } 5 \\
\text { anos, da zona } \\
\text { urbana e rural } \\
\text { do município } \\
\text { de Gameleira, } \\
\text { Pernambuco } \\
\quad(\mathrm{n}=697)\end{array}$ & $\begin{array}{l}\text { - Peso } \\
\text { e estatura }\end{array}$ & $\begin{array}{l}\text { - Indices E/I, P/ } \\
\text { I, IMC/I, em } \\
\text { escore Z } \\
\left(\mathrm{OMS}^{32}\right)\end{array}$ & $\begin{array}{l}-88,2 \% \text { dos } \\
\text { domicílios em IA } \\
-75,1 \% \text { dos } \\
\text { menores de } 5 \\
\text { anos } \\
\text { encontravam-se } \\
\text { em famílias com } \\
\text { IAM ou IAG }\end{array}$ & $\begin{array}{l}\text { - Associação entre IA, em seus } \\
\text { diferentes graus de intensidade, e o } \\
\text { índice } \mathrm{E} / \mathrm{I}(\mathrm{p}=0,01) \text {, com a média de } \\
\text { escore } \mathrm{Z} \text { diminuindo à medida que a } \\
\text { IA se agravava }\end{array}$ \\
\hline $\begin{array}{l}\text { Oliveira et } \\
\text { al. } 2009^{20}\end{array}$ & $\begin{array}{c}\text { Crianças } \\
\text { menores de } 5 \\
\text { anos, da zona } \\
\text { urbana e rural } \\
\text { de São João do } \\
\text { Tigre, semi- } \\
\text { árido do } \\
\text { Nordeste } \\
(\mathrm{n}=558)\end{array}$ & $\begin{array}{l}\text { - Peso } \\
\text { - Estatura, em } \\
\text { duplicata }\end{array}$ & $\begin{array}{l}\text { - Índices: } \\
\text { IMC/I, E/I, P/I, } \\
\text { em escore Z } \\
\left(\mathrm{OMS}^{32} ; \text { Brasil }^{33}\right)\end{array}$ & $\begin{array}{l}\text { - 86,4\% dos } \\
\text { domicílios em } \\
\text { IA, na zona } \\
\text { urbana } \\
\text { - 88,5\% dos } \\
\text { domicílios em IA } \\
\text { na zona rural } \\
\text { - 64,3\% dos } \\
\text { menores de } 5 \\
\text { anos } \\
\text { encontravam-se } \\
\text { em famílias com } \\
\text { IAM ou IAG }\end{array}$ & $\begin{array}{l}\text { - Associação entre IA e o índice } E / I \text {, } \\
\text { ( } \mathrm{p}=0,02) \text {, com média de escore } Z \\
\text { diminuindo à medida que a IA se } \\
\text { agravava } \\
\text { - O efeito da (in) segurança alimentar } \\
\text { não apresentou significância } \\
\text { estatística após ajuste para as variáveis } \\
\text { renda e escolaridade materna }\end{array}$ \\
\hline $\begin{array}{l}\text { Oliveira et } \\
\text { al. } 2009^{12}\end{array}$ & $\begin{array}{l}\text { Adolescentes e } \\
\text { adultos dos } \\
\text { municípios de } \\
\text { Gameleira e } \\
\text { São João do } \\
\text { Tigre, Nordeste } \\
(\mathrm{n}=1.528 \\
\text { adolescentes e } \\
1.163 \text { adultos })\end{array}$ & $\begin{array}{l}\text { - Peso e } \\
\text { estatura, em } \\
\text { duplicata } \\
\text { - Gestantes e } \\
\text { nutrizes não } \\
\text { foram incluídas }\end{array}$ & $\begin{array}{l}\text { - Adolescentes e } \\
\text { adultos: IMC } \\
\left(\mathrm{OMS}^{31}\right)\end{array}$ & $\begin{array}{l}-88 \% \text { dos } \\
\text { domicílios em IA }\end{array}$ & $\begin{array}{l}\text { - Ausência de associação entre (in) } \\
\text { segurança alimentar e estado } \\
\text { nutricional }(p>0,05) \\
\text { - Maior prevalência de baixo peso } \\
\text { entre adolescentes em IAM ou IAG } \\
\text { - Maior prevalência de excesso de peso } \\
\text { entre adultos em SA ou IAL }\end{array}$ \\
\hline $\begin{array}{l}\text { Pimentel } \\
\text { et al. } \\
2009^{19}\end{array}$ & $\begin{array}{l}\text { Crianças de } 6 \text { a } \\
30 \text { meses de } \\
\text { Duque de } \\
\text { Caxias, Rio de } \\
\text { Janeiro } \\
(\mathrm{n}=402)\end{array}$ & $\begin{array}{l}\text { - Peso e } \\
\text { estatura, em } \\
\text { duplicata }\end{array}$ & $\begin{array}{l}\text { - Índices: P/I, E/ } \\
\text { I, P/E, em } \\
\text { escore } \mathrm{Z}\left(\mathrm{OMS}^{32}\right)\end{array}$ & $\begin{array}{l}\text { - } 72 \% \text { das } \\
\text { famílias com } \\
\text { crianças de } 6 \text { a } \\
30 \text { meses de } \\
\text { idade em IA } \\
\text { - 12\% das } \\
\text { famílias em IAG }\end{array}$ & $\begin{array}{l}\text { - Associação negativa entre IA e os } \\
\text { índices } \mathrm{P} / \mathrm{I}(\mathrm{p}=0,01) \text { e } \mathrm{P} / \mathrm{E}(\mathrm{p}=0,05)\end{array}$ \\
\hline
\end{tabular}

*Em todos os estudos a situação de (in) segurança alimentar foi avaliada pela Escala Brasileira de Insegurança Alimentar (EBIA). PNDS: Pesquisa Nacional de Demografia e Saúde; OMS: Organização Mundial de Saúde; SA: segurança alimentar; IA: insegurança alimentar; IAL: insegurança alimentar leve; IAM: insegurança alimentar moderada; IAG: insegurança alimentar grave; IAMG: insegurança alimentar moderada/grave; P/I: peso/idade; E/I: estatura/idade; IMC: índice de massa corporal; IMC/I: IMC/idade. 
Quadro 2. Relação entre consumo alimentar e presença de insegurança alimentar domiciliar, detectada pela Escala Brasileira de Insegurança Alimentar (EBIA) nos estudos revisados.

\begin{tabular}{|c|c|c|c|c|}
\hline \multirow[b]{2}{*}{ Referência } & \multicolumn{2}{|r|}{ Metodologia } & \multicolumn{2}{|r|}{ Resultados } \\
\hline & $\begin{array}{l}\text { Unidade } \\
\text { de Estudo }\end{array}$ & $\begin{array}{c}\text { Avaliação do } \\
\text { consumo alimentar }\end{array}$ & $\begin{array}{l}\text { Insegurança } \\
\text { Alimentar }^{*}\end{array}$ & Associação entre variáveis \\
\hline $\begin{array}{c}\text { Gomes e } \\
\text { Gubert } \\
2012^{24}\end{array}$ & $\begin{array}{c}\text { Crianças } \\
\text { menores de } \\
2 \text { anos, } \\
\text { participantes } \\
\text { da PNDS, } \\
2006 \\
(\mathrm{n}=1.635)\end{array}$ & $\begin{array}{l}\text { - Analisado consumo } \\
\text { alimentar da criança no dia } \\
\text { anterior à entrevista, } \\
\text { incluindo o consumo de leite } \\
\text { materno. }\end{array}$ & $\begin{array}{l}\text { - } 47,2 \% \text { das } \\
\text { crianças do } \\
\text { estudo residiam } \\
\text { em domicílios } \\
\text { em situação de } \\
\text { IA. }\end{array}$ & $\begin{array}{l}\text { - Maior prevalência }(41,3 \%) \text { de aleitamento } \\
\text { materno entre as crianças de } 12 \text { a } 24 \text { meses em } \\
\text { situação de insegurança alimentar domiciliar } \\
(\mathrm{p}<0,05) \\
\text { - A condição de insegurança alimentar não } \\
\text { associou-se com a prática de aleitamento } \\
\text { materno no primeiro ano de vida, bem como } \\
\text { com a introdução precoce de alimentação } \\
\text { complementar neste mesmo estudo }\end{array}$ \\
\hline $\begin{array}{l}\text { Antunes et } \\
\text { al. } 2010^{23}\end{array}$ & $\begin{array}{c}\text { Crianças } \\
\text { menores de } \\
3 \text { anos de } \\
\text { Duque de } \\
\text { Caxias, Rio } \\
\text { de Janeiro } \\
(\mathrm{n}=402)\end{array}$ & $\begin{array}{l}\text { - } 2 \text { recordatórios de } 24 \text { horas } \\
\text { - Estimativa do consumo } \\
\text { (média) de energia, macro e } \\
\text { micronutrientes e de grupos } \\
\text { de alimentos } \\
\text { - Grupos de alimentos } \\
\text { determinados segundo } \\
\text { metodologia do Ministério da } \\
\text { Saúde }{ }^{34} \text { e porções de acordo } \\
\text { com Phillipi et al. }{ }^{35} \\
\text { - Adequação de energia e } \\
\text { micronutrientes avaliada de } \\
\text { acordo com o Instituto de } \\
\text { Medicina dos EUA, conforme } \\
\text { sexo e faixa etária e a dos } \\
\text { macronutrientes conforme } \\
\text { Ministério da Saúde }\end{array}$ & $\begin{array}{l}\text { - } 72 \% \text { dos } \\
\text { domicílios } \\
\text { em IA }\end{array}$ & $\begin{array}{l}\text { - Maior inadequação de consumo de proteínas } \\
\text { entre crianças em IAMG }(16,6 \%) \text { e IAL }(8,9 \%) \\
\text { ( } \mathrm{p}<0,001) \\
\text { - Consumo acima da recomendação para } \\
\text { glicídios maior em crianças em IAL }(7,9 \%) \text { e } \\
\text { IAMG }(7,6 \%) \text { ( }<0,05) \\
\text { - Maior consumo das porções dos grupos das } \\
\text { hortaliças e doces/ açúcares e menor consumo } \\
\text { de cereais e café entre crianças em IAL em } \\
\text { relação às crianças em AS (p < 0,05) } \\
\text { - Consumo reduzido de porções de cereais, } \\
\text { hortaliças, frutas, carnes/ ovos e gorduras em } \\
\text { crianças em IAMG (p }<0,05) \\
\text { - Consumo de café maior entre crianças em } \\
\text { IAMG (p < 0,05) } \\
\text { - Consumo excessivo de energia ( } 44,4 \%) \text { e } \\
\text { deficiente de ferro }(64,3 \%) \text { maior entre as } \\
\text { crianças em IAMG ( } \mathrm{p}=0,001 \text { ) } \\
\text { - Consumo de verduras/ legumes e de leite/ } \\
\text { derivados aquém das recomendações, } \\
\text { independente da presença de IA nos domicílios }\end{array}$ \\
\hline
\end{tabular}

continua

sença de filtro de água ${ }^{13,19}$, ao contrário do estudo de Souza et al. ${ }^{28}$ que encontrou associação entre insegurança alimentar e presença de água tratada no domicílio.

\section{Discussão}

Mensurar a (in)segurança alimentar e nutricional representa um desafio devido à complexidade e ao aspecto multifacetado associados a esta situação ${ }^{37}$, além do fato da EBIA ser uma escala de mensuração da presença de insegurança alimentar, não contemplando a dimensão nutricio- nal desta situação, tão relevante e já incluída no conceito de segurança adotado no país.

O estudo de Hoffmann ${ }^{38}$, analisando os dados da Pesquisa Nacional por Amostras de Domicílios (PNAD) de 2004, encontrou prevalência de 34,9\% dos domicílios com algum grau de insegurança alimentar, valor este inferior ao encontrado nos estudos incluídos nesta revisão. Este mesmo estudo demonstrou maior prevalência de insegurança alimentar na zona rural, assim como nos estudo de Oliveira et al. ${ }^{20}$ e VelásquezMelendez et al. ${ }^{26}$. Acredita-se que a maior presença de insegurança alimentar na zona rural, detectada pela EBIA, se deve à estreita dependên- 
Quadro 2. continuação

\begin{tabular}{|c|c|c|c|c|}
\hline \multirow[b]{2}{*}{ Referência } & \multicolumn{2}{|r|}{ Metodologia } & \multicolumn{2}{|r|}{ Resultados } \\
\hline & $\begin{array}{l}\text { Unidade } \\
\text { de Estudo }\end{array}$ & $\begin{array}{c}\text { Avaliação do } \\
\text { consumo alimentar }\end{array}$ & $\begin{array}{l}\text { Insegurança } \\
\text { Alimentar }\end{array}$ & Associação entre variáveis \\
\hline $\begin{array}{l}\text { Panigassi } \\
\text { et al. } \\
2008^{22}\end{array}$ & $\begin{array}{l}\text { Famílias da } \\
\text { zona } \\
\text { urbana de } \\
\text { Campinas, } \\
\text { São Paulo, } \\
\text { com pelo } \\
\text { menos um } \\
\text { morador } \\
\text { menor de } \\
18 \text { anos } \\
(\mathrm{n}=847)\end{array}$ & $\begin{array}{l}\text { - Medido pela referência do } \\
\text { consumo do informante da } \\
\text { família (sim/não), } \\
\text { considerado uma proxy do } \\
\text { consumo familiar } \\
\text { - Análise da frequência de } \\
\text { realização diária das refeições } \\
\text { principais (desjejum, almoço } \\
\text { e jantar) baseadas na } \\
\text { referência do informante da } \\
\text { família }\end{array}$ & $\begin{array}{l}\text { - 60,5\% dos } \\
\text { domicílios } \\
\text { em IA }\end{array}$ & $\begin{array}{l}\text { - Maior omissão de refeições principais nas } \\
\text { famílias em IAMG }(\mathrm{p}<0,01) \\
\text { - Consumo menor de carne, leite e derivados, } \\
\text { frutas e verduras/legumes em famílias em IA } \\
(\mathrm{p}=0,0001) \\
\text { - Consumo menor de doces e refrigerantes em } \\
\text { famílias em IA ( } \mathrm{p}=0,0001) \\
\text { - Elevado consumo diário de alimentos } \\
\text { altamente energéticos como óleo ( } \mathrm{p}<0,001) \text {, } \\
\text { açúcar ( }<<0,05) \text { e refrigerante }(\mathrm{p}=0,0001) \\
\text { entre as famílias com algum grau de IA } \\
\text { - Não houve diferença no consumo de feijão, } \\
\text { ovos e embutidos }\end{array}$ \\
\hline $\begin{array}{l}\text { Fávaro et } \\
\text { al. } 2007^{21^{+*}}\end{array}$ & $\begin{array}{l}\text { Crianças } \\
\text { menores de } \\
5 \text { anos da } \\
\text { área } \\
\text { indígena } \\
\text { Burití, } \\
\text { Mato } \\
\text { Grosso de } \\
\text { Sul, sendo } \\
\text { sorteada } \\
\text { apenas uma } \\
\text { criança por } \\
\text { família } \\
\text { (n }=49)\end{array}$ & $\begin{array}{l}\text { - Um recordatório de } 24 \\
\text { horas, referente ao dia } \\
\text { anterior à entrevista - } \\
\text { Mensuradas as quantidades } \\
\text { consumidas de calorias, } \\
\text { proteína, vitaminas A e C, } \\
\text { cálcio e ferro } \\
\text { - Adequação do consumo } \\
\text { considerado acima de } 90 \%\end{array}$ & $\begin{array}{l}\text { - 75,5\% dos } \\
\text { domicílios } \\
\text { em IA }\end{array}$ & $\begin{array}{l}\text { - Consumo menor }(16,6 \%) \text { de frutas } \\
(\mathrm{p}=0,015) \text { e carne }(38,8 \%)(\mathrm{p}<0,05) \text { entre as } \\
\text { crianças em IAMG } \\
\text { - Consumo inadequado de calorias em } 66,6 \% \\
\text { das crianças em SA/IAL e em } 94,4 \% \text { das } \\
\text { crianças em IAMG ( }>0,05) \\
\text { - Consumo inadequado de proteínas em } 44,4 \% \\
\text { das crianças em IAMG e em } 5,6 \% \text { das com SA/ } \\
\text { IAL ( }<0,05) \\
\text { - Consumo adequado de ferro em } 61,1 \% \text { das } \\
\text { crianças em SA/IAL e } 11,1 \% \text { das crianças } \\
\text { IAMG (p = 0,001) } \\
\text { - Consumo inadequado de vitamina A em } \\
88,8 \% \text { das dietas das crianças, independente do } \\
\text { nível de IA } \\
\text { - Consumo inadequado de vitamina C e cálcio } \\
\text { em } 38,9 \% \text { e } 19,4 \% \text {, respectivamente, das dietas } \\
\text { das crianças ( } p>0,05)\end{array}$ \\
\hline
\end{tabular}

‘Em todos os estudos a situação de (in) segurança alimentar foi avaliada pela Escala Brasileira de Insegurança Alimentar (EBIA); ; Utilização de EBIA adaptada para a população de estudo; PNDS: Pesquisa Nacional de Demografia e Saúde; SA: segurança alimentar; IA: insegurança alimentar; IAL: insegurança alimentar leve; IAM: insegurança alimentar moderada; IAG: insegurança alimentar grave; IAMG: insegurança alimentar moderada/grave.

cia desses indivíduos com o meio ambiente, relatada por McGregor ${ }^{39}$, em relação ao plantio de alimentos para autoconsumo, refletida nas respostas da escala, uma vez que esta avalia a percepção do entrevistado em relação com o alimento. Além disso, a pobreza, em sua forma mais intensa, está presente no meio rural, sendo intensificada pelo baixo desenvolvimento do capital humano e fraca tendência de desenvolvimento do mesmo, menores índices de educação, contribuindo assim para maior situação de insegurança nesse meio ${ }^{39-41}$.
A situação de (in)segurança alimentar e nutricional é um marcador de desigualdades relativas ao sistema econômico ${ }^{42}$, sendo a pobreza e desigualdade social são fatores determinantes da insegurança alimentar, e por esse motivo suas estimativas podem ser utilizadas, de forma complementar, para a detecção da situação de insegurança alimentar e nutricional ${ }^{4,43}$.

Mesmo com altas prevalências de insegurança alimentar nos domicílios avaliados, a presença de desnutrição em crianças foi inferior aos desta situação, o que demonstra discordância 
Quadro 3. Relação entre indicadores sociais e presença de insegurança alimentar domiciliar, detectada pela Escala Brasileira de Insegurança Alimentar (EBIA), nos estudos revisados.

\begin{tabular}{|c|c|c|c|c|}
\hline \multirow[b]{2}{*}{ Referência } & \multicolumn{2}{|r|}{ Metodologia } & \multicolumn{2}{|r|}{ Resultados } \\
\hline & $\begin{array}{l}\text { Unidade } \\
\text { de Estudo }\end{array}$ & Indicadores sociais & $\begin{array}{c}\text { Insegurança } \\
\text { Alimentar }\end{array}$ & Associação entre variáveis \\
\hline $\begin{array}{c}\text { Aires et al. } \\
2012^{30}\end{array}$ & $\begin{array}{l}\text { Famílias } \\
\text { de pré- } \\
\text { escolares de } \\
\text { uma zona } \\
\text { rural de } \\
\text { Maranguape, } \\
\text { Ceará } \\
(\mathrm{n}=200)\end{array}$ & $\begin{array}{l}\text { - Estado civil, escolaridade do } \\
\text { responsável, trabalho fora de } \\
\text { casa, número de filhos entre } 3 \\
\text { e } 6 \text { anos, número de } \\
\text { moradores no domicílio, } \\
\text { renda familiar mensal. }\end{array}$ & $\begin{array}{l}\text { - } 88 \% \text { de IA nos } \\
\text { domicílios } \\
\text { avaliados. }\end{array}$ & $\begin{array}{l}\text { - Menor escolaridade do responsável associada à } \\
\text { presença de IA }(\mathrm{p}<0,0001) \\
\text { - Maior número de moradores no domicílio } \\
\text { associado à situação de IA no domicílio } \\
(\mathrm{p}<0,05) \\
\text { - Menor renda mensal familiar associada à } \\
\text { condição de IA }(\mathrm{p}<0,0001)\end{array}$ \\
\hline $\begin{array}{l}\text { Anschau et } \\
\text { al. } 2012^{27}\end{array}$ & $\begin{array}{c}\text { Famílias } \\
\text { beneficiárias } \\
\text { de programas } \\
\text { de } \\
\text { transferência } \\
\text { de renda, em } \\
\text { Toledo, } \\
\text { Paraná } \\
(\mathrm{n}=4.525)\end{array}$ & $\begin{array}{l}\text { - Presença de moradores } \\
\text { menores de } 18 \text { anos; número } \\
\text { de moradores por domicílio; } \\
\text { renda per capita; classe } \\
\text { econômica (A, B, C, D e E); } \\
\text { sexo, escolaridade e vínculo } \\
\text { empregatício do chefe do } \\
\text { domicílio. }\end{array}$ & $\begin{array}{l}\text { - 75\% de IA } \\
\text { nas famílias } \\
\text { beneficiárias } \\
\text { de programas } \\
\text { de transferência } \\
\text { de renda. }\end{array}$ & $\begin{array}{l}\text { - Presença de moradores menores de } 18 \text { anos } \\
\text { associado à situação de IA }(\mathrm{p}<0,01) \\
\text { - Número de moradores no domicílio maior } \\
\text { que } 5 \text { associado à presença de IA }(\mathrm{p}<0,05) \\
\text { - Menor escolaridade do chefe do domicílio } \\
\text { associada à condição de IA ( } \mathrm{p}<0,05) \\
\text { - Vínculo empregatício do chefe da família } \\
\text { associado à situação de IA ( } \mathrm{p}=0,003) \text {, sendo } \\
\text { maior presença de IA em domicílios com chefes } \\
\text { desempregados ou com trabalho informal (OR } \\
=1,87 \text {; IC: } 1,18-2,95) \\
\text { - Menor renda familiar per capita associada à } \\
\text { maior condição de IA (p }<0,0001) \\
\text { - Maior presença de IA }(\mathrm{OR}=2,88 ; \text { IC: } 1,66- \\
5,0) \text { em domicílios de classe econômica D ou E } \\
(\mathrm{p}<0,0001)\end{array}$ \\
\hline $\begin{array}{c}\text { Souza et al. } \\
2012^{28}\end{array}$ & $\begin{array}{c}\text { Famílias } \\
\text { beneficiárias } \\
\text { de programas } \\
\text { de } \\
\text { transferência } \\
\text { de renda, } \\
\text { com crianças } \\
\text { de } 2 \text { a } 6 \\
\text { anos, de } \\
\text { Viçosa, MG } \\
(\mathrm{n}=243)\end{array}$ & $\begin{array}{l}\text { - Saneamento básico, número } \\
\text { de moradores, número de } \\
\text { cômodos, escolaridade } \\
\text { materna e paterna, cor ou } \\
\text { raça do titular do benefício, } \\
\text { classificação econômica } \\
\text { segundo Associação Brasileira } \\
\text { de Empresas e Pesquisa } \\
(\mathrm{ABEP})\end{array}$ & $\begin{array}{l}-72,8 \% \text { dos } \\
\text { domicílios em } \\
\text { IA } \\
-14,8 \% \text { dos } \\
\text { domicílios } \\
\text { em IAG }\end{array}$ & $\begin{array}{l}\text { - Maior presença de IA em domicílios com } \\
\text { número de moradores superior a } 4(73,1 \%) \\
(\mathrm{p}=0,01) \\
\text { - Maior situação de IA em domicílios que } \\
\text { possuíam água tratada por filtração }(74,3 \%) \\
(\mathrm{p}<0,05) \\
\text { - Associação entre escolaridade materna } \\
\text { inferior a } 10 \text { anos e IA }(79,1 \%)(\mathrm{p}<0,01) \\
\text { - Associação entre classificação econômica e } \\
\text { presença de IA ( } \mathrm{p}=0,002) \text {, sendo mais } \\
\text { prevalente em famílias com nível econômico E } \\
(91,0 \%) \text {. }\end{array}$ \\
\hline
\end{tabular}

continua

entre estes métodos de avaliação de (in)segurança alimentar e nutricional, inclusive em áreas mais pobres $^{20}$. Cabe aqui ressaltar que a insegurança alimentar e nutricional contempla além da desnutrição o excesso de peso, ambas as situações decorrentes da alimentação inadequada, entre outros determinantes, caracterizando assim a transição nutricional ${ }^{4}$. Comum a essas duas di- mensões da insegurança alimentar, tem-se a fome oculta, que corresponde à subnutrição devido à inadequação quantitativa ou qualitativa da alimentação diária, ou ainda doenças que provocam o mau aproveitamento biológico dos alimentos ingeridos ${ }^{6,7}$.

Carvalho et al. ${ }^{44}$ revelam em seu estudo que em crianças menores de cinco anos, o processo de 
Quadro 3. continuação

\begin{tabular}{|c|c|c|c|c|}
\hline \multirow[b]{2}{*}{ Referência } & \multicolumn{2}{|r|}{ Metodologia } & \multicolumn{2}{|r|}{ Resultados } \\
\hline & $\begin{array}{l}\text { Unidade } \\
\text { de Estudo }\end{array}$ & Indicadores sociais & $\begin{array}{c}\text { Insegurança } \\
\text { Alimentar }^{*}\end{array}$ & Associação entre variáveis \\
\hline $\begin{array}{l}\text { Velásquez- } \\
\text { Melendez } \\
\text { et al. } \\
2011^{26}\end{array}$ & $\begin{array}{c}\text { Mulheres em } \\
\text { idade } \\
\text { reprodutiva, } \\
\text { participantes } \\
\text { da PNDS, } \\
2006 \\
(\mathrm{n}=10.226)\end{array}$ & $\begin{array}{l}\text { - Escolaridade, cor da pele / } \\
\text { etnia, local de residência, } \\
\text { renda familiar, idade } \\
\text { materna, tabagismo, } \\
\text { estado conjugal e região } \\
\text { geográfica. }\end{array}$ & $\begin{array}{l}\text { - 40,9\% dos } \\
\text { domicílios } \\
\text { em IA }\end{array}$ & 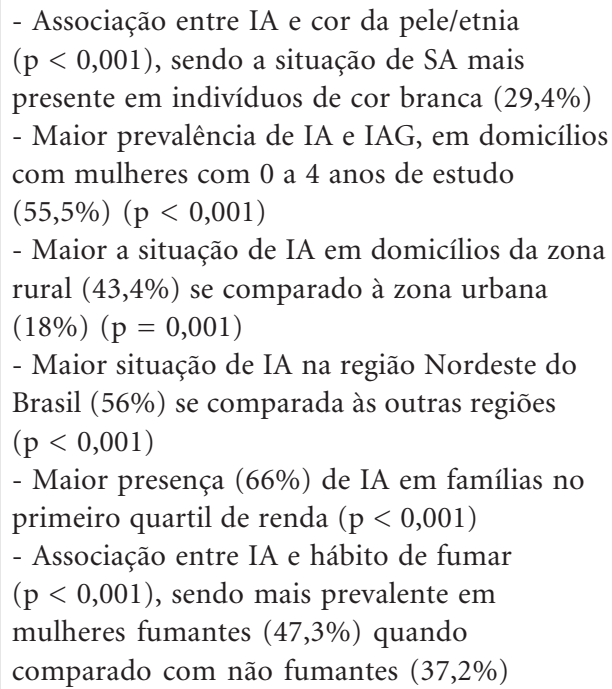 \\
\hline $\begin{array}{l}\text { Pimentel } \\
\text { et al. } \\
2009^{19}\end{array}$ & $\begin{array}{l}\text { Crianças de } \\
6 \text { a } 30 \text { meses } \\
\text { de Duque de } \\
\text { Caxias, Rio } \\
\text { de Janeiro } \\
(\mathrm{n}=402)\end{array}$ & $\begin{array}{l}\text { - Saneamento básico, } \\
\text { presença de filtro no } \\
\text { domicílio, renda familiar } \\
\text { per capita, escolaridade do } \\
\text { morador de referência da } \\
\text { família, número de } \\
\text { moradores, raça/cor da pele } \\
\text { do chefe/cônjuge da família } \\
\text { (observada), classificação } \\
\text { econômica (ABIPEME, } \\
\text { 2006). }\end{array}$ & $\begin{array}{l}-72 \% \text { das } \\
\text { famílias com } \\
\text { crianças de } 6 \text { a } \\
30 \text { meses de } \\
\text { idade em IA } \\
\text { - 12\% das } \\
\text { famílias em } \\
\text { IAG }\end{array}$ & $\begin{array}{l}\text { - Maior a situação de IA em domicílios sem } \\
\text { coleta regular de lixo }(84,5 \%)(p<0,01) \\
\text { - Maior a presença de IA em domicílios sem } \\
\text { rede pública de esgoto }(72,5 \%)(p<0,05) \\
\text { - Maior presença }(81,2 \%) \text { de IA em domicílios } \\
\text { sem filtro de água }(p<0,01) \\
\text { - Maior presença de IAMG em famílias com } \\
\text { menor renda per capita }(p<0,0001) \\
\text { - Maior situação em IA em domicílios cujo } \\
\text { morador de referência da família era analfabeto } \\
(86,9 \%) \text { ou possuía apenas ensino fundamental } \\
\text { incompleto }(84,9 \%), \text { com maior presença de } \\
\text { IAG }(p<0,0001)\end{array}$ \\
\hline
\end{tabular}

transição nutricional encontra-se bem evidente, com redução das prevalências de déficit de peso para idade e aumento do excesso de peso. Os mesmos autores destacam ainda que há diferenças significativas entre prevalências de déficit de altura para idade entre beneficiários e não beneficiários do Programa Bolsa Família, demonstrando que crianças beneficiadas apresentam, além de baixa renda familiar per capita - critério de inclusão do programa -, situação nutricional desfavorável e indicativa de presença de insegurança alimentar. Dados da PNAD de 2004 indicam que em domicílios com presença de beneficiários de programas de transferência de renda, a situação de insegurança alimentar é mais presente ${ }^{45}$.
Assim como o apresentado nesta revisão, Nobre et al. ${ }^{46}$ encontraram menor consumo de alimentos reguladores entre os indivíduos com algum grau de insegurança alimentar. O comprometimento da qualidade e quantidade dos alimentos nas famílias em insegurança alimentar resulta em um menor aporte energético e de nutrientes ${ }^{47}$, refletindo no déficit ponderal em crianças. O crescimento é ótimo indicador de desigualdade populacional, uma vez que a baixa estatura é um dos produtos da desigualdade social, permitindo inferir o déficit nutricional cumulativo $^{48,49}$. Ressalta-se que uma alimentação inadequada, muitas vezes caracterizada pelo excesso de calorias, principalmente vazias, e pelo 
Quadro 3. continuação

\begin{tabular}{|c|c|c|c|c|}
\hline \multirow[b]{2}{*}{ Referência } & \multicolumn{2}{|r|}{ Metodologia } & \multicolumn{2}{|r|}{ Resultados } \\
\hline & $\begin{array}{l}\text { Unidade } \\
\text { de Estudo }\end{array}$ & Indicadores sociais & $\begin{array}{c}\text { Insegurança } \\
\text { Alimentar }\end{array}$ & Associação entre variáveis \\
\hline $\begin{array}{c}\text { Panigassi } \\
\text { et al. } \\
2008 b^{25}\end{array}$ & $\begin{array}{c}\text { Famílias } \\
\text { residentes na } \\
\text { zona urbana } \\
\text { de } \\
\text { Campinas, } \\
\text { São Paulo, } \\
\text { com pelo } \\
\text { menos um } \\
\text { morador } \\
\text { menor de } \\
18 \text { anos } \\
(\mathrm{n}=456)\end{array}$ & $\begin{array}{l}\text { - Sexo e escolaridade do } \\
\text { chefe da família; raça/cor } \\
\text { autorreferida do informante; } \\
\text { renda familiar mensal; } \\
\text { saneamento básico; tipo de } \\
\text { habitação; referência de } \\
\text { criança com verminose no } \\
\text { domicílio; escolaridade dos } \\
\text { membros da família; } \\
\text { aglomeração domiciliar. }\end{array}$ & $\begin{array}{l}-60,5 \% \text { das } \\
\text { famílias com } \\
\text { presença de } \\
\text { menores de } 18 \\
\text { anos em } \\
\text { situação de IA. }\end{array}$ & 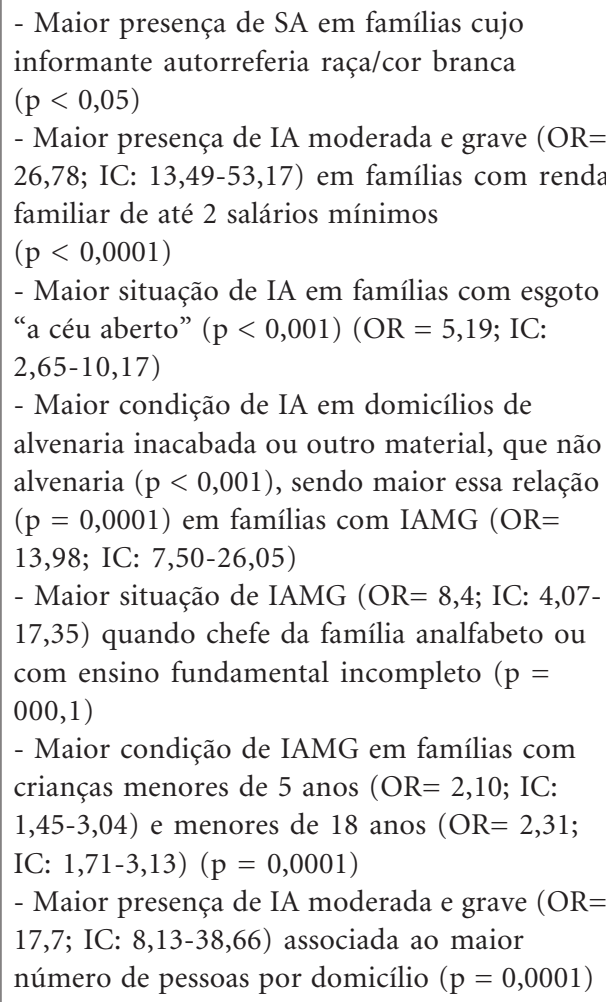 \\
\hline $\begin{array}{c}\text { Salles- } \\
\text { Costa et al. } \\
2008^{11}\end{array}$ & $\begin{array}{c}\text { Crianças } \\
\text { (6 a } 30 \\
\text { meses), } \\
\text { adolescentes } \\
\text { (12 a } 18 \\
\text { anos) e } \\
\text { adultos de } \\
\text { Duque de } \\
\text { Caxias, Rio } \\
\text { de Janeiro } \\
\text { (n = 1085 } \\
\text { domicílios) }\end{array}$ & $\begin{array}{l}\text { - Saneamento básico, renda } \\
\text { familiar per capita mensal, } \\
\text { número de pessoas por } \\
\text { domicílio, escolaridade do } \\
\text { chefe da família, classificação } \\
\text { econômica (ABIPEME) }\end{array}$ & $\begin{array}{l}-53,8 \% \text { dos } \\
\text { domicílios em } \\
\text { IA. } \\
-16,1 \% \text { dos } \\
\text { domicílios em } \\
\text { IAM e } 6,3 \% \\
\text { em IAG. }\end{array}$ & $\begin{array}{l}\text { - Maior situação de IA }(88 \%) \text { em famílias com } \\
\text { até } 1 / 4 \text { de SM per capita mensal ( }<<0,0001) \\
\text { - Maior presença de IA }(59,9 \%) \text { em domicílios } \\
\text { de chefes analfabetos ou ensino fundamental } \\
\text { incompleto (p }<0,01) \\
\text { - Associação entre nível econômico e IA } \\
\text { (p }<0,0001) \text {, sendo mais presente a IA em } \\
\text { domicílios classificados em D }(71,1 \%) \text { ou E } \\
(68,8 \%) \\
\text { - Maior presença de IA em domicílios com } \\
\text { número de filhos superior a } 5 \text { (p }<0,0001) \\
\text { - Maior situação }(63,3 \%) \text { de IA em domicílios } \\
\text { sem filtro de água (p }<0,01) \text {, se comparado a } \\
\text { domicílios com filtro de água }(48,7 \%)\end{array}$ \\
\hline
\end{tabular}

desequilíbrio de nutrientes também caracteriza a situação de insegurança alimentar e nutricional.

Segundo dados da PNAD, a insegurança alimentar moderada ou grave é mais prevalente em domicílios de baixa renda mensal per capita, localizados na zona rural, com presença de pelo menos um morador menor de 18 anos, com in- divíduos de baixa escolaridade e cor autorreferida não branca ${ }^{45,50}$. Estes achados vão ao encontro dos estudos incluídos nesta revisão sistemática, que apresentaram relação entre situação de insegurança alimentar, em seus diferentes níveis e estes indicadores socioeconômicos e demográficos ${ }^{11,19,21,22,25-29}$. 
Quadro 3. continuação

\begin{tabular}{|c|c|c|c|c|}
\hline \multirow[b]{2}{*}{ Referência } & \multicolumn{2}{|r|}{ Metodologia } & \multicolumn{2}{|r|}{ Resultados } \\
\hline & $\begin{array}{l}\text { Unidade } \\
\text { de Estudo }\end{array}$ & Indicadores sociais & $\begin{array}{c}\text { Insegurança } \\
\text { Alimentar }\end{array}$ & Associação entre variáveis \\
\hline $\begin{array}{l}\text { Vianna e } \\
\text { Segall- } \\
\text { Correa } \\
2008^{29}\end{array}$ & $\begin{array}{c}\text { Famílias } \\
\text { urbanas e } \\
\text { rurais do } \\
\text { interior da } \\
\text { Paraíba } \\
(\mathrm{n}=4.533)\end{array}$ & $\begin{array}{l}\text { - Construção da moradia, } \\
\text { saneamento básico, renda per } \\
\text { capita. }\end{array}$ & $\begin{array}{l}-52,5 \% \text { de IA } \\
\text { nos domicílios } \\
\text { avaliados. }\end{array}$ & $\begin{array}{l}\text { - Maior situação de IA em domicílios } \\
\text { construídos por material que não alvenaria (OR } \\
=1,98 \text {; IC: } 1,64-2,38) \\
\text { - Maior presença de IA em domicílios sem água } \\
\text { encanada (OR = 1,38; IC: } 1,15-1,65) \\
\text { - Maior condição de IA associada à menor } \\
\text { renda per capita nos domicílios (OR=2,18; IC: } \\
1,45 \text { - } 3,28)\end{array}$ \\
\hline $\begin{array}{l}\text { Fávaro et } \\
\text { al. } 2007^{21^{* *}}\end{array}$ & \begin{tabular}{|} 
Crianças \\
menores de \\
5 anos da \\
área indígena \\
Burití, Mato \\
Grosso de \\
Sul, sendo
\end{tabular} & $\begin{array}{l}\text { - Renda mensal per capita, } \\
\text { escolaridade materna, } \\
\text { número de filhos, número } \\
\text { de membros na família }\end{array}$ & $\begin{array}{l}\text { - 75,5\% dos } \\
\text { domicílios } \\
\text { em IA }\end{array}$ & $\begin{array}{l}\text { - Maior presença de IAMG }(73,1 \%) \text { em } \\
\text { domicílios com número de membros maior } 5 \\
\text { (OR = 3,53; IC: } 1,07-11,67) \\
\text { - Maior situação de IAMG }(73,1 \%) \text { em } \\
\text { domicílios com escolaridade materna de } 0 \text { a } 3 \\
\text { anos (p < 0,05) } \\
\text { - Maior situação de IAMG }(69,2 \%) \text { em } \\
\text { domicílios com número de filhos superior a } 3 \\
\text { (OR = 5,14; IC: } 1,52-17,38) \\
\text { - Maior presença de IAMG }(88,5 \%) \text { em } \\
\text { domicílios com renda per capita até } 1 / 4 \text { de SM } \\
\text { (OR = 7,03; IC 1,64-30,11) }\end{array}$ \\
\hline
\end{tabular}

*Em todos os estudos a situação de (in) segurança alimentar foi avaliada pela Escala Brasileira de Insegurança Alimentar (EBIA); ${ }^{* *}$ Utilização de EBIA adaptada para a população de estudo; IA: insegurança alimentar; IAMG: insegurança alimentar moderada/grave; SM: salário mínimo. OR: odds ratio; IC: intervalo de confiança.

Observou-se algumas limitações, nos estudos incluídos nesta revisão sistemática, como a pouca exploração da relação entre (in)segurança alimentar e estado nutricional, proposta no objetivo dos estudos que analisaram estas variáveis; alguns estudos não utilizaram o índice IMC/ I para crianças e adolescentes, mesmo tendo sido aceitos para publicação após indicação de referência da $\mathrm{WHO}^{32,51}$; avaliação do consumo alimentar por diferentes metodologias, e ausência de citação da tabela de composição de alimentos utilizada, o que dificulta a comparação entre os resultados dos estudos; e agrupamento das situações de segurança alimentar com insegurança alimentar leve e de insegurança alimentar moderada com insegurança alimentar grave, em alguns estudos, ou até mesmo agrupamento das três situações de insegurança alimentar. Outro fator observado foi que alguns estudos não discutem os indicadores sociais, apenas os apresentam, mesmo quando há associação deste com a insegurança alimentar.

Ressalta-se ainda que no estudo de Fávaro et al. ${ }^{21}$ utilizou-se escala de insegurança alimentar adaptada para sua população, composta por crianças indígenas, e que a mesma apresentou confiabilidade satisfatória (alfa de Cronbach de $0,93)$. Outro aspecto limitante se deve ao fato de todos os estudos incluídos nesta revisão sistemática serem transversais, impossibilitando inferências mais abrangentes, bem como relações causais entre os determinantes de (in)segurança alimentar ${ }^{52}$.

\section{Considerações Finais}

A situação de (in)segurança alimentar, determinada pela EBIA, apresentou concordância com indicadores antropométricos, dietéticos e sociais nos estudos incluídos nesta revisão. Esta escala é útil para estimar a prevalência de insegurança alimentar, em seus diferentes níveis e para identificar os grupos populacionais de risco, desde que sejam utilizados conjuntamente outros indicadores apropriados.

Medir a insegurança alimentar domiciliar é um desafio em função da complexidade e do extenso número de fatores associados a este fenômeno. Assim sendo, nenhum indicador, isolada- 
mente, consegue abranger as múltiplas dimensões da (in)segurança alimentar e nutricional.

Ainda são escassas as publicações relacionando a (in)segurança alimentar mensurada pela EBIA e indicadores do estado nutricional, consumo alimentar e indicadores, no país, indicando a necessidade de mais estudos que busquem elucidar a presença ou não de associação entre a situação de (in)segurança alimentar e outras variáveis de interesse.

\section{Colaboradores}

DC Morais trabalhou na concepção, redação final e revisão; LV Dutra, SCC Franceschini e SE Priore na redação final e revisão crítica. 


\section{Referências}

1. Brasil. Lei $n^{\circ} 11.346$, de 15 de setembro de 2006. Cria o Sistema Nacional de Segurança Alimentar e Nutricional - SISAN com vistas em assegurar o direito humano à alimentação adequada e dá outras providências. Diário Oficial da União 2006; 18 set.

2. Maluf RSJ. Definindo segurança alimentar e nutricional. In: Maluf RSJ, organizador. Segurança alimentar e nutricional. Petrópolis: Vozes; 2007. p. 17-19.

3. Barreto SM, Pinheiro RO, Sichieri R, Monteiro CA, Batista Filho B, Schimidt MI, Lotufo P, Assis AM, Guimarães V, Recine EGIG, Victora CG, Coitinho D, Passos VMA. Análise da estratégia global para alimentação, atividade física e saúde da Organização Mundial de Saúde. Epidemiol Serv Saude 2005; 14(1):41-68.

4. Sicoli JL. Pactuando conceitos fundamentais para a construção de um sistema de monitoramento da SAN. Instituto Pólis, São Paulo, 2005. [acessado 2012 jul 25]. Disponível em: http://www.polis.org.br/ download/65.pdf.

5. Segall-Corrêa AM. Insegurança alimentar medida a partir da percepção das pessoas. Estud Av 2007; 21(60):143-154.

6. Monteiro CA. A dimensão da pobreza, da fome e da desnutrição no Brasil. Estud Av 1995; 9(24):195207.

7. Maluf RS. Segurança alimentar e fome no Brasil 10 anos da Cúpula Mundial de Alimentação. Centro de Segurança Alimentar e Nutricional. Rio de Janeiro: CERESAN; 2006. (Relatórios técnicos, n. 2 CERESAN).

8. Food and Agriculture Organization of the United Nations (FAO). The State of food insecurity in the world. Economic growth is necessary but not sufficient to accelerate reduction of hunger and malnutrition. Rome: FAO; 2012.

9. Segall-Corrêa AM, Marin-Léon L, Helito H, PérezEscamilla R, Santos LPM, Paes-Sousa R. Transferência de renda e segurança alimentar no Brasil: análise dos dados nacionais. Rev Nutr 2008; 21(Supl.):39-51.

10. Melgar-Qinonez H, Hackett M. Measuring household food security: the global experience. Rev Nutr 2008; 21(Supl.):27-37.

11. Salles-Costa R, Pereira RA, Vasconcellos MTL, Veiga GV, Marins, VMR, Jardim BC, Gomes FS, Sichieri R. Associação entre fatores socioeconômicos e insegurança alimentar: estudo de base populacional na Região Metropolitana do Rio de Janeiro, Brasil. Rev Nutr 2008; 21(Supl.):99-109.

12. Oliveira JS, Lira PIC, Veras ICL, Maia SR, Lemos MCC, Andrade SLLS, Viana Junior MJ, Pinto FCL, Leal VS, Batista Filho M. Estado nutricional e insegurança alimentar de adolescentes e adultos em duas localidades de baixo índice de desenvolvimento humano. Rev Nutr 2009; 22(4):453-465.

13. Segall-Corrêa AM, Marin-Léon L. A segurança alimentar no Brasil: Proposição e usos da escala brasileira de medida da insegurança alimentar de 2003 a 2009. Rev. Segurança Alimentar e Nutricional 2009; 16(2):1-19.
14. Drewnowski A, Specter SE. Poverty and obesity: the role of energy density and energy costs. Am J Clin Nutr. 2004; 79(1):6-16.

15. Monteiro CA. A dimensão da pobreza, da desnutrição e da fome no Brasil. Estud Av 2003; 17(48):7-20.

16. Pérez-Escamilla R. Seguridad Alimentaria Y Nutricional: Marco Conceptual. In: XII Congresso Brasileiro de Sociologia, 2005, Belo Horizonte. Sociologia e realidade: pesquisa social no século XXI, 2005.

17. Campbell CC. Food insecurity: a nutritionaloutcome or a predictor variable? J Nutr 1991; 121(3):408415.

18. Oliveira JS, Lira PIC, Maia SR, Sequeira LAS, Amorim RCA, Batista Filho M. Insegurança alimentar e estado nutricional de crianças de Gameleira, zona da mata do Nordeste brasileiro. Rev. Bras. Saude Matern. Infant 2010; 10(2):237-245.

19. Pimentel PG, Sichieri R, Salles-Costa R. Insegurança alimentar, condições socioeconômicas e indicadores antropométricos em crianças em região metropolitana do Rio de Janeiro/Brasil. Rev Bras Est Pop 2009; 26(2):283-294.

20. Oliveira JS, Lira PIC, Andrade SLLS, Sales AC, Maia SR, Batista Filho M. Insegurança alimentar e estado nutricional de crianças de São João do Tigre, no semi-árido do Nordeste. Rev Bras Epidemiol 2009; 12(3):413-423.

21. Fávaro T, Ribas DLB, Zorzatto JR, Segall-Corrêa AM, Panigassi G. Segurança alimentar em famílias indígenas Terena, Mato Grosso do Sul, Brasil. Cad. Saude Publica 2007; 26(8):1642-1650.

22. Panigassi G, Segall-Corrêa AM, Marin-León L, Pérez-Escamilla R, Maranha LK, Sampaio MFA. Insegurança alimentar intrafamiliar e perfil de consumo de alimentos. Rev Nutr 2008; 21(Supl.):135-144.

23. Antunes MM, Sicheri R, Salles-Costa R. Consumo alimentar de crianças menores de três anos residentes em área de alta prevalência de insegurança alimentar domiciliar. Cad Saude Publica 2010; 23(4): 785-793.

24. Gomes GP, Gubert MB. Aleitamento materno em crianças menores de 2 anos e situação domiciliar quanto à segurança alimentar e nutricional. J Pediatr 2012; 88(3):279-282.

25. Panigassi G, Segall-Corrêa AM, Marin-León L, Pérez-Escamilla R, Sampaio MFA, Maranha LK. Segurança alimentar como indicador de iniquidade: análise de inquérito populacional. Cad. Saude Publica 2008; 24(10):2376-2384.

26. Valásquez-Melendez G, Schlussel MM, Brito AS, Silva AAM, Lopes-Filho J, Kac G. Mild but not light or severe food insecurity is associated with obesity among brazilian women. J Nutr 2011; 141(5):898-902.

27. Anschau FR, Matsuo T, Segall-Corrêa AM. Insegurança alimentar entre beneficiários de programas de transferência de renda. Rev Nutr 2012; 25(2):177189. 
28. Souza NN, Moura e Dias M, Sperandio N, Franceschini SCC, Priore SE. Perfil socioeconômico e insegurança alimentar e nutricional de famílias beneficiárias do Programa Bolsa Família no município de Viçosa, Estado de Minas Gerais, Brasil, em 2011: um estudo epidemiológico transversal. Epidemiol Serv Saude 2012; 21(4):655-662.

29. Vianna RPT, Segall-Corrêa AM. Insegurança alimentar das famílias residentes em municípios do interior do estado da Paraíba, Brasil. Rev Nutr 2008; 21(Supl.):111-122.

30. Aires JS, Martins MC, Joventino ES, Ximenes LB. (in)segurança alimentar em famílias de pré-escolares de uma zona rural do Ceará. Acta Paul Enferm 2012; 25(1):102-108.

31. World Health Organization (WHO). Physical Status: the use and interpretation of anthropometry. Geneva: WHO; 1995. (WHO Technical Report Series n. 854$)$.

32. World Health Organization (WHO). WHO Child Growth Standards: Length/height-for-age, weight-forage, weight-for-lenght, weighth-for-height and body mas index-for-age. Methods and development. Geneva: WHO; 2006. WHO (nonserial publication).

33. Brasil. Ministério da Saúde (MS). Coordenação Geral de Política de Alimentação e Nutrição. Vigilância Alimentar e Nutricional: SISVAN. Orientações para a coleta e análise de dados antropométricos em serviços de saúde. Brasília: MS; 2008.

34. Brasil. Ministério da Saúde (MS), Organização PanAmericana da Saúde. Secretaria de Políticas de Saúde. Guia alimentar para crianças menores de dois anos. Brasília: MS, Organização Pan-Americana da Saúde; 2002.

35. Phillipi ST, Cruz ATR, Colluci ACA. Pirâmide alimentar para crianças de 2 a 3 anos. Rev Nutr 2003; 16(1):5-19.

36. Ministério da Saúde (MS), Organização Pan-Americana da Saúde. Secretaria de Políticas de Saúde. Guia alimentar para crianças menores de dois anos. Brasília: MS, Organização Pan-Americana da Saúde; 2006.

37. Albuquerque MFM. A segurança alimentar e nutricional e o uso da abordagem de direitos humanos no desenho das políticas públicas para combater a fome e a pobreza. Rev Nutr 2009; 22(6):895-903.

38. Hoffmann R. Determinantes da Insegurança Alimentar no Brasil: Análise dos Dados da PNAD de 2004. Rev Segurança Alimentar e Nutricional 2008; 15(1):49-61.

39. Costa AM. Pobreza e vulnerabilidade de agricultores familiares de Santo Cristo/RS: uma análise da seca a partir da abordagem das capacitações [tese]. Porto Alegre: Universidade Federal do Rio Grande do Sul; 2006.

40. Lang RMF, Almeida CCB, Taddei JAAC. Segurança alimentar e nutricional de crianças menores de dois anos de famílias de trabalhadores rurais Sem Terra. Cien Saude Colet 2011; 16(7):3111-3118.
41. Mondini L, Rosa TE, Gubert MB, Sato GS, Benício MHD. Insegurança alimentar e fatores sociodemográficos associados nas áreas urbana e rural do Brasil. Informações Econômicas 2011; 41(2):52-60.

42. Barroso GS, Sichieri R, Salles-Costa R. Fatores associados ao déficit nutricional em crianças residentes em uma área de prevalência elevada de insegurança alimentar. Rev. Brasileira de Epidemiologia 2008; 11(3):484-494.

43. Segall-Corrêa AM, Marin-Leon L, Helito H, PérezEscamilla R, Santos LMP, Paes-Sousa R. Transferência de renda e segurança alimentar no Brasil: análise dos dados nacionais. Rev. Nutr. 2008; 21(Supl.):39-51.

44. Carvalho AT, Almeida ER, Nilson EAF, Ubarana JA, Fernández IM, Immink M. Métodos de análise em programas de segurança alimentar e nutricional: uma experiência no Brasil. Cien Saude Colet 2013; 18(2):309-321.

45. Instituto Brasileiro de Geografia e Estatística (IBGE). Pesquisa Nacional por Amostra de Domicílios - PNAD - Segurança Alimentar: 2004. Rio de Janeiro: IBGE; 2006.

46. Nobre LN, Murta NMG, Souza MM, Ferreira NC, Cardoso LM, Hamacek FR. Segurança Alimentar em uma Comunidade Rural no Alto Vale do Jequitinhonha/MG. Rev Segurança Alimentar e Nutricional 2009; 16(1):18-31.

47. Matheson DM, Varady J, Varady A, Killen JD. Household food security and nutritional status of Hispanic children in the fifth grade. Am J Clin Nutr 2002; 76(1):210-217.

48. Onis M, Frongillo EA, Blõssner N. Is malnutrition declining? As analysis of changes in levels of child malnutrition since 1980. Bull Word Health Organ 2000; 78(10):1222-1233.

49. World Bank. World development indicators. Washington: The World Bank; 2004.

50. Instituto Brasileiro de Geografia e Estatísticas (IBGE). Pesquisa Nacional por Amostra de Domicílios (PNAD). Segurança Alimentar: 2009. Rio de Janeiro: IBGE; 2010.

51. Onis M, Onyango AW, Borghi E, Siyam A, Nishida C, Siekmann J. Development of a WHO growth reference for school-aged children and adolescents. Washington: Bulletin of the World Health Organization; 2007.

52. Cotta RMM, Machado JC. Programa Bolsa Família e segurança alimentar e nutricional no Brasil: revisão crítica da literatura. Rev Panam Salud Publica 2013; 33(1):54-60.

Artigo apresentado em 31/07/2013

Aprovado em 29/09/2013

Versão final apresentada em 07/10/2013 